\title{
THE EFFECT OF X-RAY RADIATION ON THE HUMAN BODY - PROS AND CONS. RADIATION PROTECTION IN MEDICAL IMAGING AND RADIOTHERAPY
}

\author{
Zhenya Zhekova-Maradzhieva ${ }^{1}$, Bistra Velchovska ${ }^{1}$, Atanas Uzunov ${ }^{1}$, Evgeniya Ivanova ${ }^{2}$, \\ Desislava Petrova ${ }^{3}$, Mariana Yordanova ${ }^{4}$, Georgi Valchev ${ }^{5}$ \\ ${ }^{1}$ Student, TRS X-Ray Laboratory Assistant, Medical College, Medical University of Varna \\ ${ }^{2}$ Student, TRS Assistant Pharmacist, Yordanka Filaretova Medical College, \\ Medical University - Sofia \\ ${ }^{3}$ Student, TRS Assistant Pharmacist, Medical College, Medical University of Varna \\ ${ }^{4}$ TRS X-Ray Laboratory Assistant, Medical College, Medical University of Varna \\ ${ }^{5}$ Department of Radiology, St. Marina University Hospital, Varna
}

\begin{abstract}
INTRODUCTION: The discovery of X-rays in November 1895 by Roentgen opened a new chapter in the scientific development and pretty soon it became clear that these rays can be useful for diagnostics and treatment. The most frequent use of X-rays is related to their ability to pass through matter. The main fields of application of the rays are medicine, industry, checks of goods and packages and scientific studies. Modern medicine constitutes approximately $80 \%$ of the overexposure. The contribution of diagnostic radiology is approximately $22 \%$ of the total exposure of Bulgarian population. The quality of the medical services depends to a great extent on the accurate and timely diagnoses set through different methods using also ionizing radiation. The exposure of the patient should be reasonably justified and optimized but cannot be limited. The risk of exposure to high doses of radiation is justified only if this is appropriate for the diagnosis or the treatment. Each overexposure, including medical irradiation, is related to certain radiation risk. Radiation protection is a means to apply the measures intended to protect the health from ionizing radiation-related risks. It is essential to know the benefits and risks of the medical procedures.
\end{abstract}

AIM: To investigate radiation protection means, and the benefits and risks of medical procedures.

MATERIALS AND METHODS: An analysis of literature sources was conducted.

RESULTS: The medical control of the radiation protection divides exposure into three categories: professional exposure, medical exposure and exposure of the population. Irradiation by any source should be conducted by optimizing the protection and the safety, maintaining the size of the individual dose, the number of exposed persons and the exposure at levels as low as reasonably achievable considering the social and economic factors. This is the so-called optimization of the protection.

Address for correspondence:

Zhenya Zhekova-Maradzhieva

TRS X-ray Laboratory Assistant

Medical College

Medical University of Varna

84 Tsar Osvoboditel Blvd

9000 Varna

e-mail:jeni_mar@abv.bg
CONCLUSION: The deterministic effect for the staff can be reduced through the introduction of practical actions aimed at decreasing the dose. It is recommendable that the procedures be performed safely for the staff and for the patient.

Keywords: exposure, control, radiation protection, safety 
The Effect of X-Ray Radiation on the Human Body - Pros and Cons. Radiation Protection in Medical Imaging ...

\section{INTRODUCTION}

The sources of radiation are: cosmic rays - $14 \%$ of the natural exposure, radioactive elements in the soil and in construction materials - $20 \%$ of the natural exposure, air - $51 \%$ of the natural exposure, food and water - $15 \%$ of the natural exposure (1). Depending on the place of residence, the annual effective exposure dose varies between 1 and $10 \mathrm{mSv}$, the average annual value received by the Bulgarians being 2.33 $\mathrm{mSv}$.

Wilhelm Conrad Röntgen, born in 1845, discovered a new type of rays which were invisible until that moment (13). This happened in 1895. After discovering the "X-rays" it became clear (13) that they can be useful to humans but they can also be harmful. Apart from diagnostics, the scientists started applying the new rays for treatment as well (13).

\section{AIM}

The aim of this paper is acknowledging the risks and benefits of diagnostic and/or treatment exposure, and providing information on the main principles and measures of radiation protection.

\section{MATERIALS AND METHODS}

We have used a documentary method. The authors have researched and analyzed literature sources and normative documents, regulation principles, norms and measures on radiation protection as well as on the impact of X-ray radiation on the human body.

\section{PRESENTATION}

Soon after the discovery of X-rays it was established that in addition to being useful (18) they can also be harmful (16). With time solid knowledge in the field of medicine, radiology, radiation protection, radiobiology is being accumulated. The skillful use of this knowledge leads to optimal results in the use of X-rays. Radiological treatment is used for different purposes but its medical application (18) is associated the most with a direct effect on human health. In medicine, X-rays are still used (13) for medical imaging and radiotherapy. Other types of radiations as the ionizing rays (20) are also used. They possess different energies. Radiotherapy too often depends on the accurate and timely diagnosis established with the help of different diagnostic methods.
In order to evaluate the radiation risk from the radiation impact, the following terms have been introduced:

$\diamond$ The absorbed dose, which is the proportion of the average imparted energy by ionizing radiation in an elementary unit of the irradiated matter to the mass of the substance in that unit. It is measured in Grays (Gy). Provided the absorbed dose is identical, the radiobiological effect depends on the type of the radiation and its energy. The radiation weighting factor measures the degree of harmful impact of the different radiations. For X-rays, gamma-rays and electrons the radiation weighting factor equals to 1 and for alpha rays it is $20(1,13)$.

$\diamond$ The equivalent dose is the average absorbed dose for every organ or tissue and multiplied by the relevant radiation weighting factor. It is measured in Sieverts (Sv) $(1,13)$.

The tissue weighting factor measures the relative contribution of every organ or tissue in the total impairment of the body (13). Its values are different for the different organs and tissues.

$\diamond$ The effective dose is the result of multiplying the equivalent dose by the relevant tissue weighting factor. The resulting values for all tissues and organs are then summed. It measures the summarized effect of radiation on the body. The measurement unit is Sievert (Sv) (13).

Ionizing radiations $(20,17)$ are those where the impact on the organic or non-organic matter leads to the formation of electrical loads of different charges - in short they ionize the substance. They could be corpuscular - an elementary particle flow of different mass, with or without electrical charge (alphaand beta-particles (5), neutrons), and electromagnetic (photon) radiation: gamma- and x-rays. They are also classified by: distribution speed, range in matter, density and ionization type.

$\mathrm{X}$-ray radiation appears to slow down the speed of electrically charged particles (mostly electrons). At $\mathrm{X}$-ray impact on the substance, three main processes occur: photoelectrical effect (1), Compton effect and an electron-positron pair is formed. Initially the atoms are ionized, but afterwards a secondary ionization of the separate charged particles (electrons and 
Zhenya Zhekova-Maradzhieva, Bistra Velchovska, Atanas Uzunov et al.

positrons) occurs, and gamma radiation originating from the impact on the atomic nuclei.

The biological impact of ionizing radiation depends on the total absorbed dose, the dose concentration, the type of the radiation, the volume of the irradiated surface (19), the time and the character of the exposition, the accumulation of the substance in a given organ or system, the type and individual sensitivity.

The impact on the human body comes as a result of external and/or internal exposure (1). The biological effect (7) of the external exposure is assessed based on the penetrating capacity of ionizing radiation. Human injuries are classified into stochastic (probable) and nonstochastic (deterministic) effects $(9,7)$. Stochastic effects are in a linear relationship with the absorbed dose and are considered as not having any threshold values $(6,7)$, while nonstochastic effects appear when a certain threshold value of the radiation dose is exceeded.

\section{Deterministic (nonstochastic) $(9,6)$}

These are effects that have a threshold value (8) and which appear at lethal-dose radiation (5) of a big number of cells causing irreversible injuries of the affected organ or system as well as of the entire body (13). Such effect is the acute and chronic radiation sickness, radiation skin burns, eye cataract and reduced life duration (10). Nonstochastic effects $(9,7)$ are observed in cases of exposure with absorbed dose above 1-2 Gy, and increase with increasing the dosage (13). Approximately $200 \mathrm{mGy}$ are sufficient for a presentation of mental impairment in cases of exposure during the second or third month of the pregnancy that is why it is recommended to terminate the irradiation.

\section{Stochastic (probable) $(9,6)$}

A risk of late stochastic effects appears at equivalent dose up to $200 \mathrm{mSv}$ (13). Their probability depends on the absorbed dose and on the radiation sensitivity of the irradiated tissue. (13) The presentations can be radiation carcinogenesis, malformations and carcinogenesis in the next generations. The stochastic effects $(9,7)$ also include the impacts on the cellular gene apparatus. They can also be expressed by developing leukemia and other lympho- and hemoblastoses, malignant new formations (thyroid gland cancer, osteosarcomas etc.), anomalies in the development of the fetus (in cases of exposure during pregnancy). The latent period for the development of malignant new formations is $10-25$ years. A highly probable late effect of the ionizing radiation is the genetic impact on the gametes leading to changes in heredity (19).

Radiation risk depends on the irradiated organ or tissue (13), on the type of the radiation and its energy (1). The different organs have different sensitivity to radiation.

The use of ionizing radiation for medical purposes in Bulgaria is regulated (1) by: a law, regulations (30;0-33; 0-34) and ordinances (Ordinance on the Main Radiation Protection Norms - 2004). A "dose threshold value" has been established, and it cannot be exceeded when irradiating certain persons (1) for a certain period of time as a result of performing controllable activity. The term "dose threshold" is exact as it determines the following: up to that point and this level shall be exceeded in no case (1). The main principles of radiation protection are: justification of every exposure, optimization of the protection and thresholds of the dose. These principles are applied both for the exposure of staff and patients. Radiation protection is a means to exercise the applied measures for protecting the health against risks associated with sources of ionizing radiation. This protection aims at excluding the occurrence of deterministic effects and at reducing the probability of stochastic (probable) ones (13).

It is important for patients to know what the risks of the medical procedures are and that nobody is going to take better care of us except us alone.

Radiation protection is based on three main principles:

$\diamond$ justification of every activity related to ionizing radiation: benefit - risk analysis;

$\diamond$ optimization of the protection;

$\diamond$ reducing the exposure through introducing dose thresholds.

These principles should be applied both to the staff and the patients.

Radiation protection of staff also includes additional requirements for equipment, rooms, and personal protective equipment.

10 Golden Rules for Radiation Protection of the Staff: 
The Effect of X-Ray Radiation on the Human Body - Pros and Cons. Radiation Protection in Medical Imaging ...

1. Use personal protection equipment like:

$\diamond$ lead apron;

$\diamond$ lead glasses for protecting the eyes;

$\diamond$ lead collar for thyroid gland protection.

2. Use effectively:

$\diamond$ reduction of the exposure time;

$\diamond$ increase of the maximum possible distance, as the dose decreases as per the distance quadrant;

$\diamond$ screening through effective use of stationary, mobile and individual shields (screens);

$\diamond$ decrease of the quantity of scattered radiation through blending the radiation field - decrease of the exposure for both the patient and the staff.

Each of these factors decreases the dose for the radiology practitioner from 2 to 20 times!!!

3. Use protective screens, protective shades, side and mobile protective shields, especially while performing a scopic procedure.

4. Avoid hand contact with the shaft of radiation rays as it leads to an increase of the exposure dose.

5. The main source of exposure for the radiology practitioner is the scattered radiation from the patient. Quite a small part of the rays falling on the patient pass on the other side, the rest are absorbed by their body. Thus, the patient is a source of scattered radiation, which increases the dose for the staff.

6. The X-ray tube should be below the patient table and not above it.

7. It is obligatory to use a personal dosimeter.

8. Constant update of the knowledge in the field of radiation protection is recommended.

9. Discuss the issues of radiation protection with experts in the field.

10. Control the quality of the equipment in order to guarantee safe and stable work. Know the properties of the equipment. Correct use of its capacities allows decreasing the dose both for the staff and the patient (15).

10 Golden Rules for Radiation Protection of the Patients during Scopic Procedures

1. Increase as much as possible the distance between the X-ray tube and the patient.
2. Decrease the distance between the patient and the image converter.

3. Decrease the time needed to perform a scopic procedure! Record the time needed to perform a scopic procedure and the DAP/KAP value (if available) for every patient.

4. Use the lowest possible speed for pulsed scopy where the images have an acceptable diagnostic quality.

5. Avoid the irradiation of the same area of the skin when using different projections.

6. Examining patients with fuller figure or thicker parts of the body requires an increase in the entrance skin dose (ESD).

7. Curved projections also increase the ESD. It should not be forgotten that increasing ESD also increases the probability of skin burn!

8. Avoid the use of magnification modes of the scopic device. Decreasing the surface of the visual field twice increases the dose power 4 times.

9. Decrease the number of recorded images and videos to the acceptable clinical level.

10. Use a blending tool! (14)

During radiography the skin dose is always lower because the time is also less compared to that for radioscopy.

Radiotherapy should be applied (10) only for malignant new formations (cancer) and exceptionally for non-oncologic diseases (arthroses, skin diseases, thyrotoxicosis etc.). The objective of the exposure is destroying the tumor and saving the life of the patient. The implemented total focal dose (TFD) reaches up to $80 \mathrm{~Gy}$. This inevitably leads to exposure of the surrounding healthy tissues and protective measures should be taken to avoid acute radiation impairments and to avoid late somatic and genetic effects as is the case for radiological and nuclear medical diagnostics. That is why the protective measures during radiotherapy are part of the individual plan.

Radiotherapy for non-tumor formations is applied only if there is no other option.

The opportunities for decreasing the doses during radiotherapy are:

$\diamond$ The application of the technique that is suitable for a certain patient. 
Zhenya Zhekova-Maradzhieva, Bistra Velchovska, Atanas Uzunov et al.

$\diamond$ Radiotherapy should be applied only by highly qualified specialists.

$\diamond$ Radiotherapy for non-oncological diseases should be limited.

\section{CONCLUSION}

The imaging methods using ionizing radiation are broadly applied in medical diagnostics and therapy. Radiation protection of the patients and the staff working with ionizing radiation in medical imaging is regulated by the respective legislative documents. The procedures should be implemented accurately and safely in order to maximally reduce the deterministic and stochastic effects. The deterministic effect for the staff can be reduced through the introduction of practical actions aimed at decreasing the dose. It is recommended that the procedures be performed safely for the staff and for the patient.

\section{REFERENCES}

1. Statev S. Medical radiation physics. Veliko Tarnovo: Advertising agency "MAREA DESIGN"; 2006. 215-303.

2. Ordinance on radiation protection for activities involving the use of sources of ionizing radiation. Decree of the Council of Ministers No200/2007.

3. Regulation No.33 on the design and structure of the medical radiological cabinets and on the exploitation of medical radiological systems of maximum voltage up to $300 \mathrm{Kw}$

4. Lazarov R, Borisova R, Mihaylova I, Gesheva N. Opportunities of high-technology radiotherapy of lung tumors. 2010; 12. Available at: http://www. medinfo.bg/spisanie/2010/12/statii/vyzmojnosti-navisokotehnologichnoto-lychelechenie-pri-tumorina-belija-drob-1037

5. Todorov V, Konstantinov B, Buchakliev Z, Popits R, Hadzhieva T. National conference on physics training "Physics in biology and medicine". Radiotherapy in Bulgaria and the medical physicist in it. Yambol, 6-9 April 2006. Available at: http:// roentgen-bg.org/files/3.1.\%20Radiotherapy-text.pdf

6. Deterministic And Stochastic Effects.

Available at: http://ozradonc.wikidot.com/ deterministic-and-stochastic-effects

7. Deterministic effects and stochastic effects. Available at: http://www.hko.gov.hk/education/ dbcp/rad_health/eng/r4_1.htm
8. Wang D, Jones J. Deterministic effects. Available at: https://radiopaedia.org/articles/ deterministic-effects

9. Deterministic vs. stochastic models. Available at: http://www4.stat.ncsu.edu/ gross/BIO560\%20webpage/slides/Jan102013.pdf

10. https://en.wikipedia.org/wiki/Radiation_therapy

11. https://en.wikipedia.org/wiki/Stochastic radiation therapy,

12. http://medpedia.framar.bg

13. http://www.rentgen.free.bg/index.html

14. https://rpop.iaea.org/RPOP/RPoP/Content/ Documents/Whitepapers/poster-patient-radiationprotection-bg.pdf

15. https://rpop.iaea.org/RPOP/RPoP/Content/ Documents/Whitepapers/poster-staff-radiationprotection-bg.pdf

16. Radiation Protection, Updated Thursday, August 27, 2015. Available at: http://www.nrc.gov/aboutnrc/radiation.html

17. Radiotherapy. Available at: http://medicaldictionary.thefreedictionary.com/radiotherapy

18. http://www.nrc.gov/about- nrc/radiation/relatedinfo.html

19. Side effects of radiotherapy. Available at: http:// www.nhs.uk/Conditions/Radiotherapy/Pages/Sideeffects.aspx

20. Stochastic radiation effect. Available at: https:// www.euronuclear.org/info/encyclopedia/s/stochastic-radiation-effect.htm 